\title{
THE MEMS HAMMER, A TOOL TO STUDY MICROFRACTURE
}

\author{
J. Greenspun, T. Massey, and K.S.J. Pister \\ University of California at Berkeley, USA \\ Berkeley Sensor and Actuator Center
}

\begin{abstract}
A MEMS actuator, dubbed the MEMS hammer, capable of storing and rapidly releasing mechanical energy has been designed, built and tested. The hammer is fabricated using a single mask silicon-on-insulator (SOI) process. These devices have been used to study fracture in both lateral and vertical regimes. The lateral tests have shown excellent agreement with shear fracture theory. Using either a mechanical or an electrostatic latching mechanism, the hammers are capable of storing energies up to $3.3 \mu \mathrm{J}$. The hammers have been shown to displace up to $36 \mu \mathrm{m}$, exert a maximum force of $240 \mathrm{mN}$, move at speeds exceeding $50 \mathrm{~m} / \mathrm{s}$, and deliver at least 330 $\mathrm{mW}$ of mechanical power.
\end{abstract}

\section{INTRODUCTION}

The goal of this research is to create a microactuator capable of fracturing barriers made of silicon and silicon dioxide. At its core, the MEMS hammer is a device capable of storing and releasing mechanical energy. This system could be used for applications ranging from jumping microrobots [1] to needle-free delivery of drugs and vaccines [6]. In this work, the MEMS hammer is used as a tool to study fracture at the microscale. Additionally, we show promising steps towards creating a self-destructing sensor.

MEMS devices are commonly used in energy harvesting applications to transduce mechanical energy into electrical energy, often using piezoelectric materials [11]. However, limited work has been done at the microscale to store energy in a mechanical state. One way to store energy mechanically is in tension in a beam. The maximum energy stored in a beam of length $l$, cross-sectional area $A$, and Young's Modulus $\mathrm{E}$ is given by the following equation where $\epsilon_{\max }$ is the maximum strain the material can undergo before fracture

$$
E_{\max }=\frac{1}{2} F_{\max } x_{\max }=\frac{1}{2} A l E \epsilon_{\max }^{2}
$$

Bergbreiter et al $[1,2]$ have successfully stored and released $100 \mathrm{uJ}$ of mechanical energy using polydimethylsiloxane (PDMS) as their energy storage medium. These devices were designed to work with micromechanical motors which have an output force of $10 \mathrm{mN}$. This relatively small force was the driving factor for using an elastomer based energy storage system. To store large energies with a small applied force, large displacements had to be achievable.

Rogers et al [3] created a mechanical energy storage and rapid release system in polysilicon using Sandia's SUMMiT-V process. This device was capable of using microfabricated electrostatic motors coupled to a 20,000:1 gear reduction ratio to launch a projectile with $19 \mathrm{~nJ}$ of energy.

Both of the abovementioned systems were created with the intent of using a MEMS motor to store the initial mechanical energy, and the designs were constrained accordingly. These motors have an inherently low force output, so the researchers were forced to use a large mechanical advantage or a material with a low Young's modulus. In this research, the initial mechanical energy is stored in the device in a post fabrication assembly step, allowing us the freedom to choose a simple design and a material more suitable for microfabrication.

\section{DESIGN}

The MEMS hammer is fabricated in a single mask SOI process

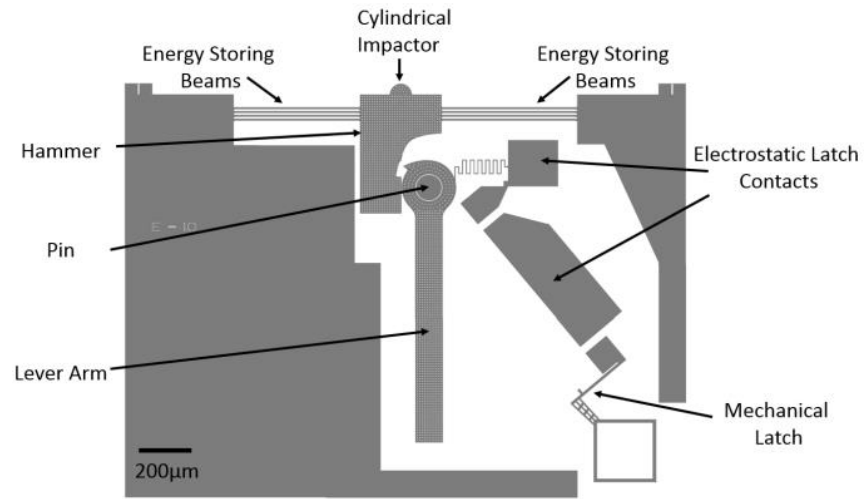

Figure 1: The layout of a MEMS hammer capable of storing $0.8 \mu \mathrm{J}$ of energy.

with a $40 \mu \mathrm{m}$ device layer, a $2 \mu \mathrm{m}$ buried oxide, and a $500 \mu \mathrm{m}$ handle wafer. The MEMS hammer, shown in Figure 1, is composed of three main components: a cylindrical impactor, energy storing beams and a latching mechanism. In standard operation, the lever arm is pushed towards the right using a probe tip. As the lever arm rotates about the pin, it catches the hammer and starts loading the beams. The mechanical latch is moved to the side to allow the lever arm to pass by. Finally, the mechanical latch is moved back into its resting position and the hammer is latched in a high energy state. A latched hammer can be seen in Figure 3b, left.

To successfully build a device capable of fracturing silicon, it is crucial to identify and reinforce all load bearing features in the design. The beams are the main energy storing elements in this device; without filleting the interface between these springs and the anchoring silicon, they will fail at deflections of less than $15 \mu \mathrm{m}$, as opposed to $40 \mu \mathrm{m}$ with the fillets.

Additionally, the pin must be able to withstand the maximum force of the springs, which can be up to $240 \mathrm{mN}$. The pin is anchored to the substrate via the buried oxide layer, and should theoretically fail when the shear stress induced by the hammer exceeds the fracture stress of the oxide. The literature on fracture stress of thinfilm silicon dioxide varies from $0.77 \mathrm{MPa}$ [12] to $364 \mathrm{MPa}$ [13]. In earlier versions of the MEMS hammer, a fracture stress of $364 \mathrm{MPa}$ was used to design these pins. Every pin tested failed catastrophically. It was determined that a pin of radius $46 \mu \mathrm{m}$ can withstand forces up to $\sim 300 \mathrm{mN}$, putting the estimated fracture stress of our oxide at $54 \mathrm{MPa}$. It is important to make this pin radius as small as possible to keep the force required to latch the hammer low. The distance from the center of the pin to the end of the lever arm divided by the distance from the center of the pin to the hammer is roughly equal to the mechanical advantage scale factor seen by the mechanical and electrostatic latches. That is to say, the smaller the pin radius is, the easier it is for these latches to restrain the hammer. Therefore, the pin radius was optimized to allow for ease of latching while still being robust enough to withstand the high forces from the hammer.

Lastly, the piece of the lever arm that catches and loads the hammer must be designed carefully. The first iterations of the hammer used square etch holes. These stress concentrations caused the lever arm to fracture at its contact point with the hammer. In the current design, this was mitigated by moving to circular etch holes. 


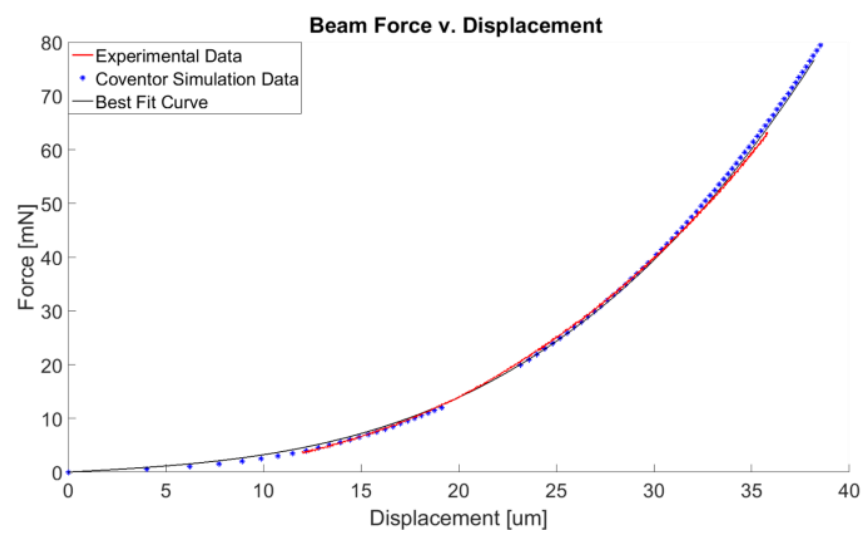

Figure 2: MEMS hammer force vs displacement data. The empirical force $v$ s displacement data is fit to the theoretical curve given by equation 5 and is in agreement with Coventor FEA.

\section{Nonlinear Beam Model}

In many MEMS applications, small displacement approximations are made to simplify calculations. These approximations are valid if the angle of beam deflection is small, such that the small angle approximations hold [4]. In this small displacement regime, the force displacement relationship of a centrally loaded fixed-fixed beam can be described using standard Euler-Bernoulli beam theory:

$$
F(x)=16 \frac{E w^{3} t}{l^{3}} x
$$

This equation accounts for the bending strain energy stored in the beam. The MEMS hammer design uses beams which are $1000 \mu \mathrm{m}$ long and deflect more than $35 \mu \mathrm{m}$ at their center. The angles reached are large enough that nonlinear beam theory must be used, which adds a third order term corresponding to the axial stretching of the beam. A simple derivation of this non-linear term is done by assuming the springs stretch linearly, like a rubber band. By ignoring the boundary conditions at the fixed and guided edges, the derivation becomes a simple geometry problem. The non-linear term can be shown to be:

$$
F_{\text {stretch }}(x)=8 \frac{E w t}{l^{3}} x^{3}
$$

Combining this with the linear force-displacement relation given by equation 2 , we arrive at the following equation which approximates the force-displacement relation of a fixed-fixed beam

$$
F(x)=16 \frac{E w^{3} t}{l^{3}} x+8 \frac{E w t}{l^{3}} x^{3}
$$

This equation corresponds to the force output for a single beam. In most hammer designs, multiple beams are used in parallel to increase the maximum force output by the device. This manifests itself in equation 5 with the addition of a factor $N$, equal to the total number of beams, in front of both terms. The integral under this force curve is equal to the energy stored by the hammer.

The energy storing characteristics of the beams used in this work were calibrated with a Dage 4000 multipurpose bondtester. This tool applies displacement at a fixed rate and measures the resulting reaction force. Figure 2 shows this data plotted against a finite element analysis (FEA) performed using Coventor. The data from the bondtester was then fit to equation 5 to determine how closely this simplified beam model describes our system. The coefficients of the best fit curve are 24.3 and 7.4 for the linear term and the third order term respectively. We see excellent agreement with our theory for the non-linear stretching term, and fairly poor agreement with the linear bending term. This is in part due to the inability of our bondtester to accurately measure forces of less than $\sim 5 \mathrm{mN}$. Figure 2 shows that not a single data point was acquired until the beams had displaced $12 \mu \mathrm{m}$, well outside the linear range of these beams. Additionally, the critical region of operation is at high displacements where the most energy is stored. In this region, we see less than $5 \%$ deviation from theory and simulation.

\section{MICROFRACTURE}

Once the energy storage and force output of the hammer are characterized, the device can be used to study microfracture. By fabricating the hammer in front of fracturable structures, we can study in-plane lateral fracture. This lateral fracture can be described by a simple shear model or a Hertzian model. With the addition of one more lithography step, we create individual hammer chiplets, shown in Figure 4, top. These chiplets allow us to study out of plane vertical fracture, and open up the possibility of interfacing the MEMS hammer with non-microfabricated structures.

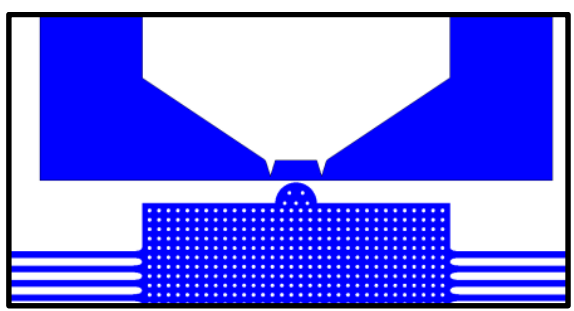

Figure 3a: A layout representation of a lateral barrier with two triangular stress concentrations. The width of the semi-circular hammer head is $40 \mu \mathrm{m}$.
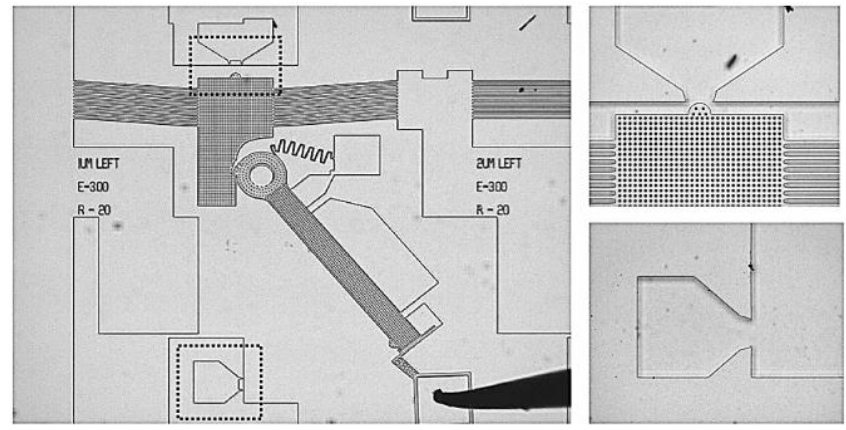

Figure 3b: A mechanically latched MEMS hammer. The mechanical latch restrains the hammer [left], until the latch is released allowing the hammer and lever arm to accelerate towards their respective barriers. Close up views of the fractured barriers for the hammer [top] and lever arm [bottom] are shown on the right.

\section{Lateral Shear Fracture}

In the lateral regime, hammers with varying stored energy are used to determine the minimum energy necessary to fracture a given barrier. Figure $3 \mathrm{~b}$ shows that each hammer contains two identical barriers, shown in the dashed boxes, that can potentially be fractured by the device. A zoomed in picture of the barrier before fracture is shown in Figure 3a. The original intent was to use the hammer itself to fracture these barriers, however once the latching mechanism was added to the design, it became apparent that the lever arm absorbs more of the stored energy than the hammer. This is explained by noting the relative distance to the pivot point of the hammer versus the end of the lever arm. This 10x difference in radius turns into a 100x difference in kinetic energy, assuming the masses are identical. 
The hammer is roughly $10 \mathrm{x}$ as massive as the end of the lever arm, so the lever arm winds up with about 10x the kinetic energy as the hammer.

The energy stored in either the hammer or the lever arm must be greater than some critical value for the barrier to fracture. A simple shear force model states that as soon as the applied force causes the induced stress in the material to exceed the fracture stress, the barrier will fail. The energy that caused this fracture event is equal to the critical force times the displacement, where the critical force is equal to the fracture stress $(1.3 \mathrm{GPa}$ [5]), times the crosssectional area $A$

$$
E_{\text {shear }}=F_{\text {crit }} x_{\max }=\sigma_{\max } A x_{\max }
$$

\section{Lateral Hertzian Fracture}

Shear theory is not the only describing physics for this lateral fracture mode. Hertzian fracture, first described by Hertz in 1881 [7], is the phenomenon in which a cone of material is ejected during a fracture event. This fracture pattern is most commonly seen in brittle amorphous materials, though it has been studied in crystalline materials as well [9].

From $[8,9]$, the Hertzian fracture criterion is described by the following equation, where $E, k$, and $v$ are material properties, and $r$ is the radius of the impactor:

$$
E_{\text {hertzian }}=\frac{2}{5}\left[\frac{2 \pi \sigma_{\max }}{1-2 v}\right]^{5}\left[\frac{4 k}{3 E}\right]^{4} r^{3}
$$

In the above equation we see the characteristic dependence on impactor radius; small-radius impactors should require less energy to produce Hertzian fracture than large-radius impactors.

\section{Vertical Fracture}

Lateral fracture is advantageous due to its simplicity in layout and ease of testing, however it limits the potential applications of the hammer. The barrier test structures must be fabricated directly in front of the hammer, and typically from the same material. With the addition of a second lithography and etch step, we can create more useful barrier structures as well as released hammer chiplets.

The reinforced membranes, shown in Figure 4, middle bottom, are simple to fabricate and straightforward to model. The structure is made of a thin circular oxide membrane with a $40 \mu \mathrm{m}$ reinforcing silicon plate at the center. We are interested in the applied force and displacement required to fracture these reinforced membranes. From Roark [10], the critical force and critical displacement required to fracture this membrane follow these proportionalities, where $a$ and $b$ are the outer and inner radii of the membrane respectively, and $t$ is the oxide thickness:

$$
\begin{aligned}
& F_{\text {crit }} \propto \frac{t^{2} b}{a} \\
& d y_{\text {crit }} \propto \frac{a^{2}}{t}
\end{aligned}
$$

Relations 8 and 9 help provide intuition for reinforced membrane fracture. The full equations are plotted in Figure 5; each curve represents a plate of different radius, swept from a $1 \mu \mathrm{m}$ oxide at the left to a $20 \mu \mathrm{m}$ oxide on the right. The required forces and displacements to fracture these membranes are well within the capabilities of the MEMS hammer. The black curve on this plot shows the maximum forces and displacements achievable by one of our hammers. The X's on this plot represent three different reinforced membranes that have been successfully fractured by our hammers.
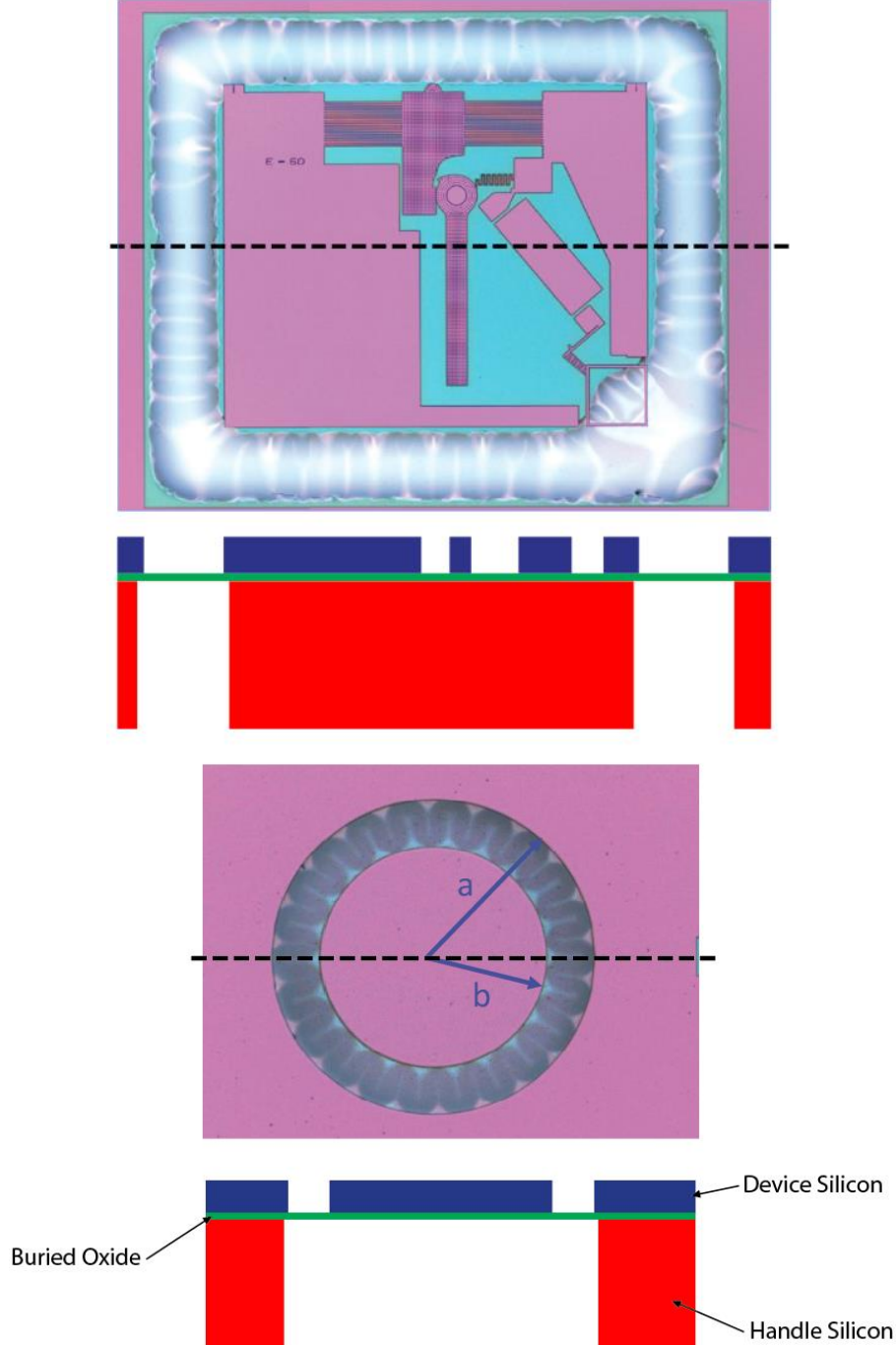

Figure 4: A 2x2 $\mathrm{mm}^{2}$ hammer chiplet before oxide release as fabricated [top] and in cross section [middle top]. A 600 $\mu \mathrm{m}$ reinforced circular membrane as fabricated [middle bottom] and in cross-section [bottom].

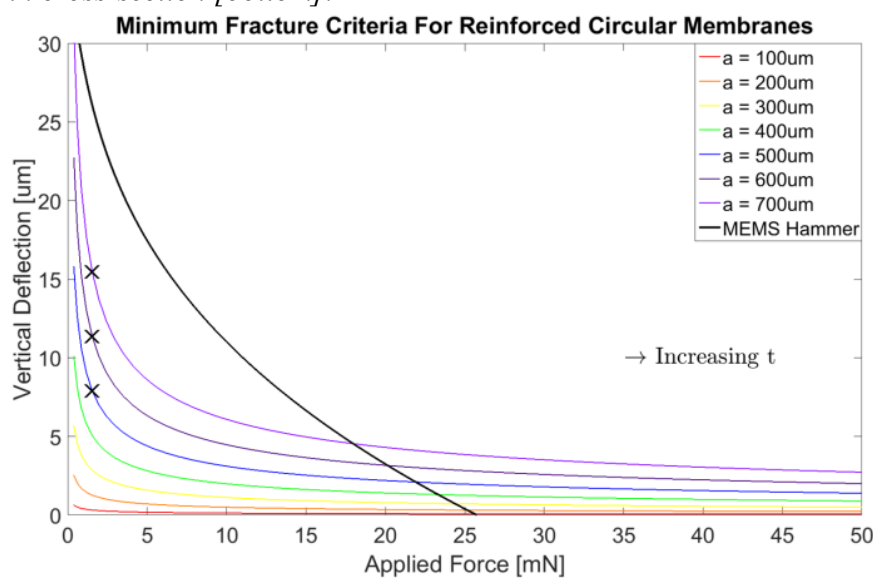

Figure 5: Reinforced circular membrane fracture theory. Each curve holds fixed the outer radius, a, while sweeping the oxide thickness, $t$, from $1 \mu \mathrm{m}$ to $20 \mu \mathrm{m}$. The black curve shows maximum forces and displacements achievable by the MEMS hammer. Black $X$ 's indicate membranes that have been fractured by hammers in testing. 


\section{RESULTS}

Arrays of hammers were fabricated with stored energies from $0.2 \mu \mathrm{J}$ to $3.3 \mu \mathrm{J}$ and cylindrical impactors of $20 \mu \mathrm{m}, 40 \mu \mathrm{m}$, and $60 \mu \mathrm{m}$ in radius. Lateral fracture studies were performed with these arrays at a probe station. Hammers were latched into place and released to test which barriers they were capable of fracturing.

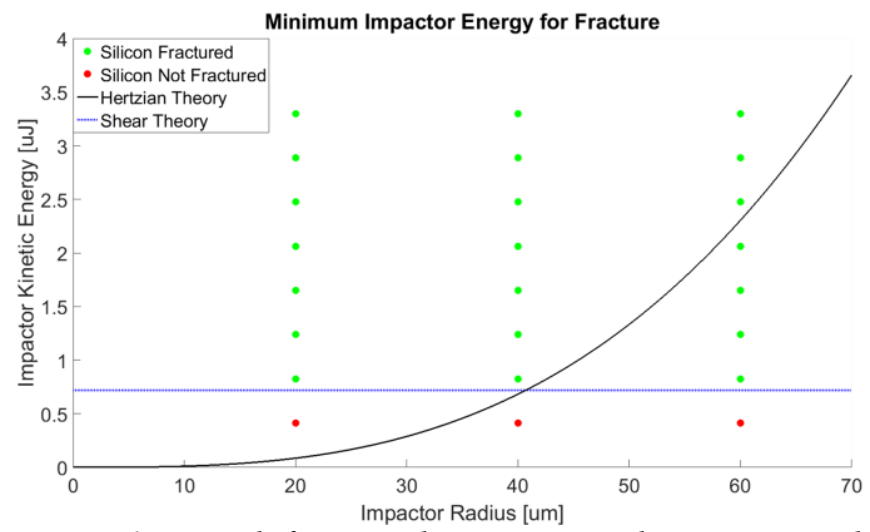

Figure 6: Lateral fracture characteristics. The superimposed Hertzian and Shear Theory lines show the minimum energy required to produce fracture in each of those regimes.

Figure 6 shows the results of the lateral fracture experiments. The two curves on the plot show the minimum energy required by the impactor to induce fracture under the two fracture theories, shear and Hertzian. It is clear from the data that this system is well described by a simple shear force analysis. The threshold for fracture was not a function of the impactor radius, as would be the case in Hertzian fracture. However, in barriers with no stress concentrations, as opposed to those with stress concentrations seen in Figure 3a, we start to see something akin to Hertzian fracture. Figure 8 shows a barrier after a $3.3 \mu \mathrm{J}$ impact from a $60 \mu \mathrm{m}$ radius lever arm. The fracture pattern on the opposite side of the barrier looks like it could be conical in form. More experiments will need to be run to determine if this fracture was in fact Hertzian, but this is a promising first result.

In the vertical fracture mode, the most robust membranes we fabricated only required an energy of $0.1 \mu \mathrm{J}$ to fracture. Every hammer we tested with these membranes fractured them easily. The weakest hammer used on the membranes was $0.8 \mu \mathrm{J}$. The test setup is shown in Figure 7. A latched MEMS hammer chiplet is picked up with tweezers and rotated until the direction of actuation is normal to the membrane surface. It should be noted that once latched, these hammers are very secure. They remained latched in place even when dropped to the floor (an all too common event during testing). The hammer is then fixed in place using a UV curable epoxy. The mechanical latch is disengaged using a probe tip and the hammer accelerates forward, breaking through the reinforced oxide membrane.

When designing an energy storage device, it is important to keep in mind the maximum theoretical energy one could store with that material. Equation 1 gives the maximum energy that can be stored in a beam. By dividing this value by the volume of that beam, and assuming the maximum strain is $1 \%$, we arrive at the maximum mechanical energy possibly stored in silicon of $8 \mathrm{~mJ} / \mathrm{mm}^{3}$. Using this figure, the total possible energy our beams could store would be $1.6 \mu \mathrm{J}$. Each beam actually stores $0.21 \mu \mathrm{J}$, just over $13 \%$ of the maximum possible energy that could be stored in this volume of silicon. When these beams are arrayed together to increase the stored energy, the gaps between adjacent springs are roughly the width of the springs, dropping the total energy storage figure to $6.5 \%$ of the theoretical maximum. After including the mass of the hammer, latch, and anchoring structures, this figure plummets to less than $1 \%$. However, these structures have not been optimized for high energy densities, and with a few simple layout modifications this number could easily be pushed to greater than $20 \%$ of the theoretical maximum.
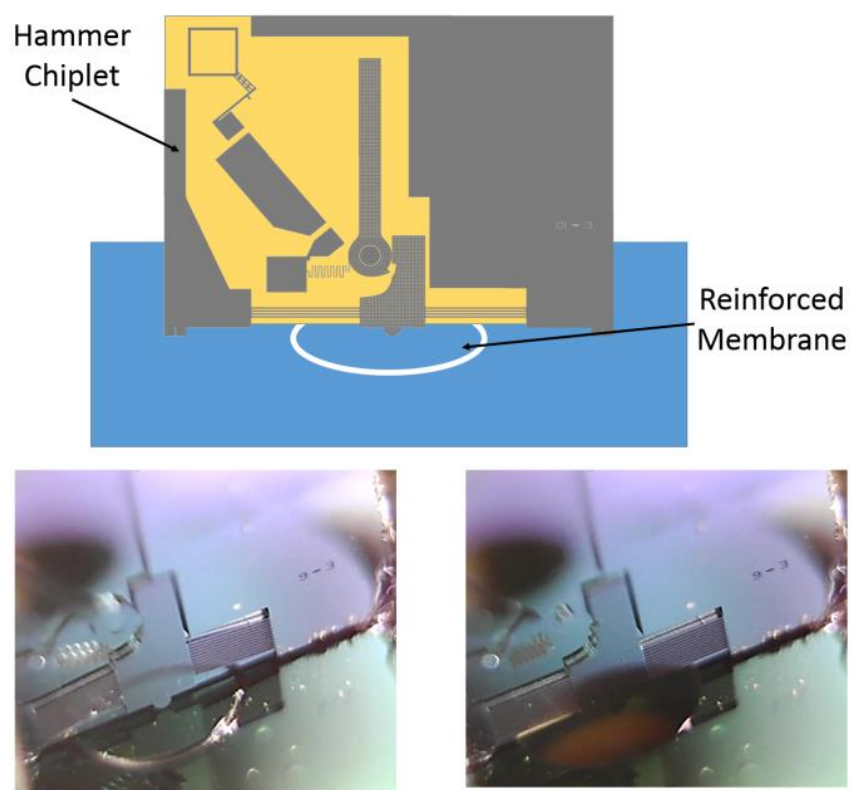

Figure 7: [Top] Cartoon version of hammer chiplet interfaced with reinforced circular membrane. Fabricated hammer and membrane before fracture [bottom left] and after fracture [bottom right].

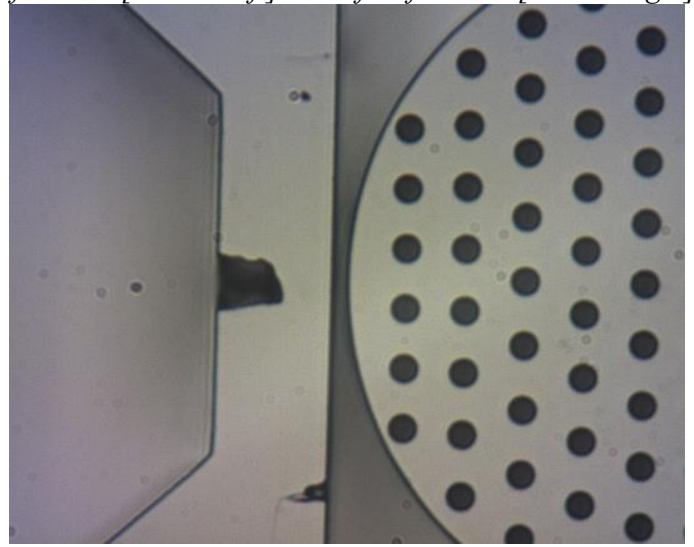

Figure 8: A potential Hertzian fracture cone. Circular etch holes are $4 \mu \mathrm{m}$ in diameter.

\section{ELECTROSTATIC LATCHING}

The use of a mechanical latch to release stored energy prohibits the MEMS hammer's use in any application outside of a lab with a probe station. To address this, we designed and tested an electrostatic latch, seen in Figure 9. This electromechanical latch relies on a relatively weak electrostatic attraction between two plates and amplifies it through one or more mechanical advantage stages. This electrostatic force is governed by the following equation, where $V$ is the applied voltage across the plates, $\epsilon_{0}$ is the vacuum permittivity, $A$ is the overlap area, and $d$ is the gap between the plates:

$$
\mathrm{F}_{\mathrm{es}}=\frac{1}{2} \epsilon_{0} \mathrm{~V}^{2} \frac{\mathrm{A}}{\mathrm{d}^{2}}
$$




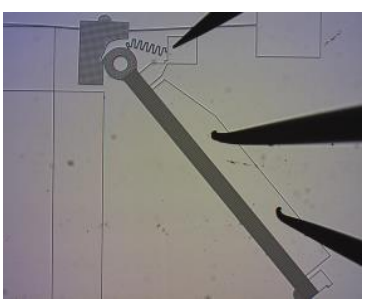

Figure 9: Single stage electrostatically latched hammer.

This electrostatic force is then multiplied by the mechanical advantage factor mentioned earlier. This mechanical advantage factor is equal to half the distance from the pin to the tip of the lever arm divided by the distance from the pin to the hammer. The factor of 0.5 shows up because this electrostatic force is distributed along the entire length of the of the lever arm. To first order, if there are multiple stages of the electrostatic latch, as can be seen in Figure 10, each stage contributes a mechanical advantage factor. The mechanical advantage factor in our system is roughly 5 , so we see an enhancement of 5 in the single stage and an enhancement of 125 in the three stage latch. Using this theory, the single stage latch should require $72 \mathrm{~V}$ to latch and the three stage latch should require $60 \mathrm{~V}$.

Figure 9 shows a very weak hammer which can store roughly $0.05 \mu \mathrm{J}$ of mechanical energy. A voltage of approximately $160 \mathrm{~V}$ was needed to close this latch. The three stage latch is shown in Figure 10. This hammer stores $3.3 \mu \mathrm{J}$ of energy, and the actual voltage required to keep the latch closed was $120 \mathrm{~V}$. Both of these devices required roughly twice the theoretical voltage to remain latched. This deviation from theory may be due to an error in the gap, which was designed to be $0.25 \mu \mathrm{m}$ but was not measured. Adding more stages than this, we believe we can design an electrostatic latch that works on as little as $5 \mathrm{~V}$.

\section{CONCLUSION AND FUTURE WORK}

We have developed and tested a MEMS device capable of storing and rapidly releasing up to $3.3 \mu J$ of energy. After simulation of the dynamics of this system, it has been shown that the device releases its energy in $10 \mu \mathrm{s}$. This means the hammer can deliver in excess of $330 \mathrm{~mW}$ of mechanical power. The hammer has been used to study fracture in both lateral and vertical regimes. The fracture of the lateral barriers was shown to be well described with a shear force model. Electrostatic latches have also been designed and verified.

The MEMS hammer is a versatile device, with target applications spanning multiple fields. This project is funded under the DARPA Vanishing Programmable Resources program. The goal of which is to create a sensor capable of complete and irreversible self-destruction. We propose using the MEMS hammer to fracture a reinforced circular membrane which covers a cavity of xenon difluoride. This powerful silicon etchant would sit dormant in an inert environment until the time comes for the sensor to destroy itself. At this point, the MEMS hammer triggers, the membrane is fractured, and the xenon difluoride is free to sublimate and attack any silicon it contacts. One additional application we are pursuing is developing a system to convert the stored mechanical energy of the hammer into electrical energy.

\section{ACKNOWLEDGEMENTS}

We would like to thank the staff of the UC Berkeley Marvell Nanolab for their help during this research project. We would also like to thank DARPA for funding this research.

\section{CONTACT}

Joey Greenspun, greenspun@eecs.berkeley.edu

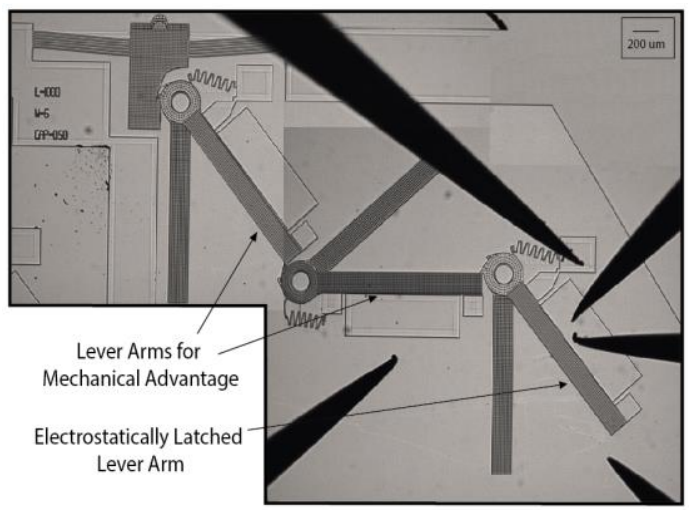

Figure 10: A three stage electrostatically latched MEMS hammer. Device too large to fit in field of view; figure stitched together from multiple images. Lever arm shadows are caused by an optional oxidation step before releasing the structures.

\section{REFERENCES}

[1] S. Bergbreiter, D. Mahajan, and K. S. J. Pister, "A reusable micromechanical energy storage / quick release system with assembled elastomers," Journal of Micromechanics and Microengineering, vol. 19, May 2009.

[2] W. Churaman, A. P. Gerratt, and S. Bergbreiter, "First leaps toward jumping microrobots," in IEEE IROS, San Francisco, CA, September 25-30, 2011.

[3] Rodgers M S, Allen J J, Meeks K D, Jensen B D and Miller S L "A micromechanical high-density energy storage/rapid release system" SPIE Conf. on Micromachined, 1999.

[4] Senturia, Stephen D. Microsystem Design. Boston: Kluwer Academic, 2001. Print.

[5] Wilson, Carol J., and Patricia A. Beck. "Fracture testing of bulk silicon microcantilever beams subjected to a side load." Microelectromechanical Systems, Journal of 5.3 (1996)

[6] Arora A, Hakim I, Baxter J, Rathnasingham R, Srinivasan R, Fletcher D A and Mitragotri S 2007 Needle-free delivery of macromolecules across the skin by nanoliter-volume pulsed microjets Proc. Natl. Acad. Sci. USA 104 pp 4255-60

[7] H. Hertz, J. Reine Angew. Math. 92, 156 (1881). Reprinted, in English, in Hertz's Miscellaneous Papers (Macmillan and Co., Ltd, London, 1896), Chap. 5.

[8] F.B. Langitan, and B.R. Lawn "Hertzian Fracture Experiments on Abraded Glass Surfaces as Definitive Evidence for an Energy Balance Explanation of Auerbach's Law,", Journal of Applied Physics, Vol. 40, No. 10, 4009-4017 (1969)

[9] B.R. Lawn "Hertzian Fracture in Single Crystals with Diamond Structure", Department of Department of Defense Advanced Research Projects Agency, 1967

[10] Roark, Raymond J., and Warren C. Young. Roark's Formulas for Stress and Strain. 6th ed. pp. 461, (1989)

[11] Cook-Chennault, K. A., N. Thambi, and A. M. Sastry. "Powering MEMS portable devices-a review of nonregenerative and regenerative power supply systems with special emphasis on piezoelectric energy harvesting systems." Smart Materials and Structures 17.4 (2008): 043001.

[12] Hatty, Veronica, Harold Kahn, and Arthur H. Heuer. "Fracture toughness, fracture strength, and stress corrosion cracking of silicon dioxide thin films." Microelectromechanical Systems, Journal of 17.4 (2008): 943-947.

[13] Sharpe Jr, W. N., et al. "Strain measurements of silicon dioxide microspecimens by digital imaging processing." Experimental Mechanics 47.5 (2007): 649-658. 